\title{
KINETICS OF FORMATION AND METASTABILITY MECHANISM FOR THERMAL DONOR-RELATED DEFECTS IN Al-DOPED SILICON*
}

\author{
P. KACZOR \\ Institute of Physics, Polish Academy of Sciences \\ Al. Lotników 32/46, 02-668 Warsaw, Poland
}

\begin{abstract}
We present a detailed investigation of the growth kinetics of aluminium-related shallow thermal donors: the $\mathrm{K}$-donors. Constraints for the diffusion mechanism of oxygen in silicon at temperatures $\approx 470^{\circ} \mathrm{C}$ are found. A large entropy of the $\mathrm{K}$-donors is considered as a possible explanation of high diffusivities and interaction radii found for the generation of the K-donors.
\end{abstract}

PACS numbers: 71.55.Cn, 66.30.Lw, 61.72.Cc

For almost 40 years, since their discovery in the 1950's [1], thermal donors (TD) in silicon are certainly among the most studied defect systems in solids. Although much effort has been put into the investigation of this defect, the microscopic structure and the generation kinetics of thermal donors are not fully understood [2]. Even less is known about the TD-related defects, shallow thermal donors (STD), observed for the first time in the mid eighties by photo-thermal ionization spectroscopy [3].

In the Al-doped samples a new member of the STD-family has been found, called the K-donor [4]. It is generated simultaneously with other shallow thermal donors. It has a very similar binding energy, but, in contrary to other STD, it exhibits a metastable behaviour. As it has been found only in Al-doped samples, it is believed to contain aluminium [4].

In this paper we present results of the generation kinetics of the $\mathrm{K}$-donor. The creation process of the donor is linked to a more general problem of oxygen diffusion in silicon at TD-annealing temperatures. We also attempt to explain the unusual metastable behaviour of the K-donors in terms of an entropy-driven transition. The large entropy change accompanying the metastable transition of the $\mathrm{K}$-donors at low temperatures can in turn be the physical reason of the very efficient diffusion processes leading to the creation of the defect.

The K-donor generation experiment was carried out with boron and aluminium doped Si samples (WASO-grade) in numerous annealing steps at $470^{\circ} \mathrm{C}$

${ }^{*}$ This work has was financially supported in part by the Committee for Scientific Research grants: No. 2P302 11705 and No. 2P302 17110. 
in the nitrogen atmosphere. The concentration of the generated species: TD, STD and K-donors was measured by means of the IR-absorption (Bomem DA3-spectrometer). The details of the photo-excitation experiment showing the metastable behaviour of the K-donor are described in [4].

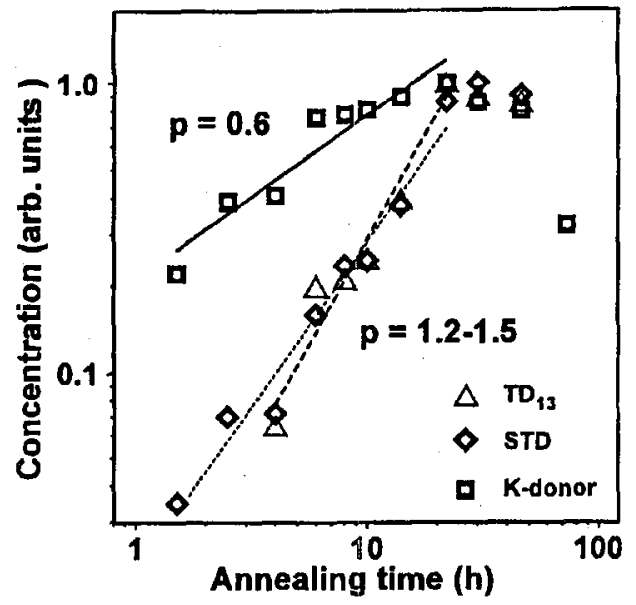

Fig. 1. The annealing time dependence of the concentrations of various oxygen agglomerates. The kinetics for the TD as well as STD obeys a power law: concentration $\sim t^{p}$, with $p=1.5-1.7$ for each of 20 donor species found. The power for the K-donor generation kinetics is twice smaller than for the other oxygen agglomerates.

The generation kinetics approximately follows the power law $n(t) \sim t^{p}$, where $t$ is the annealing time (Fig. 1). The power $p$ for all (!) TD and STD species is $1.5-1.7$ and for the K-donors $p=0.6$.

We assume that the K-donors, formed only in Al-doped samples, must contain aluminium. As the diffusivity of aluminium in silicon at the TD-annealing temperature is by three to five orders of magnitude lower than that of oxygen [5], the $\mathrm{Al}$ atoms act as quasi-unmovable seed-centres for fast diffusing oxygen atoms $\left(D=D_{\text {oxygen }}\right)$. We considered four simple reaction schemes for 1 or 2 agglomerating oxygens: (a) $\mathrm{Al}+\mathrm{O} \rightarrow \mathrm{K}$-donor (2nd order, type II), (b) $\mathrm{Al}+2 \mathrm{O} \rightarrow \mathrm{K}$-donor (3rd order, type II), (c) $\mathrm{Al}+\mathrm{O} \rightarrow$ Intermediate, Intermediate $+\mathrm{O} \rightarrow \mathrm{K}$-donor (both reactions of 2nd order, type II), (d) $\mathrm{O}+\mathrm{O} \rightarrow \mathrm{O}_{2}, \mathrm{O}_{2}+\mathrm{Al} \rightarrow \mathrm{K}$-donor (both reactions of 2 nd order, type II). Each of the schemes is described by a nonlinear differential equation (or set of them) of the first order with time dependent rate coefficients. The time-dependence of the reaction rate coefficients $k$ is characteristic of the diffusion controlled process: $k=4 \pi D R(1+R / \sqrt{\pi D t})$, where $D-$ sum of diffusivities of the reaction elements and $R$ - interaction radius [6].

Except for the general case of (d) (formation of a diffusing oxygen molecule) exact solutions depend on only 2 parameters: $R_{\mathrm{ox}}$ - the interaction radius and $D_{\text {ox }}$ - the diffusivity of the single oxygen atom ${ }^{\dagger}$. In the scheme (d) a simplifying

${ }^{\dagger} \mathrm{A}$ detailed account of the mathematical derivations will be given elsewhere. 
assumption was made: the reaction rate of the molecule formation is by a constant factor $f$ smaller than the rate for the $\mathrm{O}_{2}+\mathrm{Al} \rightarrow \mathrm{K}$-donor reaction. Then, the model of fast diffusing (faster than the isolated oxygen atoms themselves) oxygen molecule [7] can be tested. Each model was then fitted to the experimental K-donor generation kinetics and values of $R_{\mathrm{ox}}$ and $D_{\mathrm{ox}}$ were obtained. The results are summarized in Fig. 2.

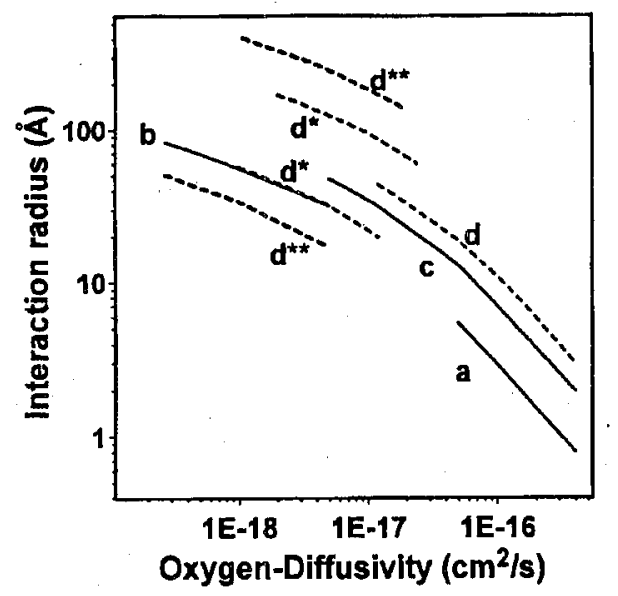

Fig. 2. The interaction radius $(R)$ vs. oxygen diffusivity $\left(D_{\mathrm{ox}}\right)$ for various K-donor agglomeration mechanisms (explanation of letter symbols can be found in the text). The lines represent $\left(R, D_{\mathrm{ox}}\right)$ pairs obtained from the best fit to the experimental K-donor annealing kinetics for the particular agglomeration mechanism. Values of $D_{\text {ox }}$ cover the range from the $D_{\mathrm{ox}} \approx 5 \times 10^{-19} \mathrm{~cm}^{2} / \mathrm{s}$, established for $T_{\text {annesl }}>600^{\circ} \mathrm{C}$ or $T_{\text {annesl }}<$ $350^{\circ} \mathrm{C}[2]$, to the value $\approx 10^{-16}$ obtained in Ref. [1].

For the model of fast diffusing oxygen molecule the $R\left(D_{\text {ox }}\right)$ curve was obtained at three different ratios of the $\mathrm{Al}+\mathrm{O}_{2}$ reaction rate to the oxygen molecule formation rate. When this ratio is greater than 1, a pair of curves is obtained - the "higher" one for the $\mathrm{Al}+\mathrm{O}_{2}$ reaction, the lower one for oxygen molecule formation. As the ratio of reaction rates increases $\left(d, d^{*}, d^{* *}\right.$ in Fig. 2) the diffusion coefficient of oxygen $-D_{\text {ox }}$ can be "reduced" to the value found at high temperatures $\left(T>500^{\circ} \mathrm{C}\right)$ but on the other hand, the interaction radii $R_{\mathrm{ox}}$ and $R_{\mathrm{O} 2}$ remain high $(\approx 70 \AA)$ or grow rapidly to $\approx 300 \AA$, respectively.

From the modelling of the K-donor formation kinetics an important conclusion can be drawn: each mechanism of the K-donor generation (one can imagine a similar behaviour for the TD-generation) based on the diffusion of oxygen (atoms or molecules) has to assume either unusually large interaction radius of $\approx 70 \AA$, when the oxygen diffusivity has its "regular" value of $5 \times 10^{-19} \mathrm{~cm}^{2} / \mathrm{s}$ [2], or unusually large diffusivity, when the interaction radius is comparable with the van der Waals radius of isolated oxygen atom $(\approx 1.5 \AA)$. Values of $D_{\text {ox }}$ by three orders of magnitude larger than the $5 \times 10^{-19} \mathrm{~cm}^{2} / \mathrm{s}$ value have been used to explain 
various results on generation and decay of TD [2]. No physical reason for that high diffusivity has been conclusively established yet.

Possibly, an answer to this problem can be found in the metastable behaviour of the $\mathrm{K}$-donors. It appears that the shallow $\mathrm{K}$-donors themselves are metastable states of deep defects exhibiting large lattice relaxation [4]. This defect forms a deep DX-like state [8] from which it can be metastably photoionized and transformed into the $\mathrm{K}$-donor state (the shallow-deep bistability). At temperatures $\approx 77 \mathrm{~K}$ (well below the critical temperature for the shallow-deep transition) this transformation allowed for any "classical" DX-like defect is inhibited. We assume that an entropy-driven transition takes place here to another deep state which cannot be photoionized [9]. The change of energy necessary to accomplish this transition was estimated to be $\approx 50 \mathrm{meV}$. The corresponding entropy change [9] is $7-8 k_{\mathrm{B}}$ which is 1.5 times larger than for any other known "simple" deep centre. We can then envisage the K-donors as an extended defect - in a sense that the lattice surrounding the defect is distorted over a large area (large configurational and/or vibrational entropy). This, in turn, can be related to the large interaction radius needed for the $\mathrm{K}$-donor formation reactions.

\section{References}

[1] C.S. Fuller, J.A. Ditzenberger, N.B. Hannay, E. Buehler, Phys. Rev. 96, 833 (1954).

[2] J. Michel, L.C. Kimmerling, in: Semiconductors and Semimetals, Vol. 42, Eds. R.K. Willardson, A.C. Beer, W.R. Weber, vol. ed. F. Shimura, Academic Press, New York, 1994, p. 251.

[3] J.A. Griffin, H. Navarro, J. Weber, L. Genzel, J.T. Borenstein, J.W. Corbett, L.C. Snyder, J. Phys. C 19, L579 (1986).

[4] P. Kaczor, K. Kopalko, M. Godlewski, T. Gregorkiewicz, Proc. 17th Intern. Conf. on Defects in Semicondictors, Gmunden (Austria) 1993, Ed. H. Heinrich, W. Jantsch, Mater. Sci. Forum, Vol. 117-120, Trans Tech Publications, Switzerland 1994, p. 1185.

[5] Landoldt-Börnstein New Series III/22b, Springer-Verlag, Berlin 1989, p. 230.

[6] M. Smoluchowski, Z. Phys. Chem. 92, 129 (1917).

[7] U. Gösele, K.-Y. Ahn, B.P.R. Marioton, T.Y. Tan, S.-T. Lee, Appl. Phys. A 48, 219 (1989).

[8] For a review see e.g. J.E. Dmochowski, L. Dobaczewski, J.M. Langer, W. Jantsch, Phys. Rev. B 40, 9671 (1989).

[9] S.T. Pantelides, Phys. Rev. B 36, 3462 (1987). 